\title{
Long-range Automaton Models of Earthquakes: Power-law Accelerations, Correlation Evolution, and Mode-switching
}

\author{
Dion Weatherley, ${ }^{1}$ Peter Mora,${ }^{2}$ and Meng Fen XiA ${ }^{3}$
}

\begin{abstract}
We introduce a conceptual model for the in-plane physics of an earthquake fault. The model employs cellular automaton techniques to simulate tectonic loading, earthquake rupture, and strain redistribution. The impact of a hypothetical crustal elastodynamic Green's function is approximated by a long-range strain redistribution law with a $r^{-p}$ dependance. We investigate the influence of the effective elastodynamic interaction range upon the dynamical behaviour of the model by conducting experiments with different values of the exponent $(p)$. The results indicate that this model has two distinct, stable modes of behaviour. The first mode produces a characteristic earthquake distribution with moderate to large events preceeded by an interval of time in which the rate of energy release accelerates. A correlation function analysis reveals that accelerating sequences are associated with a systematic, global evolution of strain energy correlations within the system. The second stable mode produces Gutenberg-Richter statistics, with near-linear energy release and no significant global correlation evolution. A model with effectively short-range interactions preferentially displays Gutenberg-Richter behaviour. However, models with long-range interactions appear to switch between the characteristic and GR modes. As the range of elastodynamic interactions is increased, characteristic behaviour begins to dominate GR behaviour. These models demonstrate that evolution of strain energy correlations may occur within systems with a fixed elastodynamic interaction range. Supposing that similar mode-switching dynamical behaviour occurs within earthquake faults then intermediate-term forecasting of large earthquakes may be feasible for some earthquakes but not for others, in alignment with certain empirical seismological observations. Further numerical investigation of dynamical models of this type may lead to advances in earthquake forecasting research and theoretical seismology.
\end{abstract}

Key words: Critical point hypothesis, cellular automata, correlation evolution.

\footnotetext{
${ }^{1}$ QUAKES, The Univ. of Queensland, Brisbane, 4072, Australia.

E-mail: weatherley@quakes.earth.uq.edu.au

${ }^{2}$ QUAKES, The Univ. of Queensland, Brisbane, 4072, Australia.

E-mail: mora@quakes.earth.uq.edu.au

${ }^{3}$ State Key Laboratory of Nonlinear Mechanics, Institute of Mechanics, Chinese Academy of Sciences, Beijing, 100080, China. E-mail: xiam@Inm.imech.ac.cn, Department of Physics, Peking University, Beijing, 100871, China.
} 


\section{Introduction}

It is well known that the regional or global distribution of earthquake magnitudes empirically obeys the Gutenberg-Richter law (GUTENBERG and RichTER, 1956), a power-law event size distribution of the form:

$$
N(M)=K_{M} M^{-b}
$$

where $K_{M}$ is an empirical constant and $b$ is the so-called regional or global $b$ value. Empirical values for the $b$ value typically fall in the range $0.8<b<1.2$. If seismic energy is employed in place of magnitude, the corresponding empirical exponent is $b \sim 2 / 3$ (Wu, 2000).

Seismologists became interested in cellular automata as possible analogues of earthquake fault dynamics when BAK and TANG (1989) demonstrated that even highly simplified, nearest neighbour automata produce power-law event size distributions. The sandpile automaton of BAK et al. (1987) was employed to demonstrate the emergence of power-law statistics as the result of the combined action of a large number of simple elements which interact only with nearby cells via a prespecified interaction rule. BAK et al. (1987) termed such behaviour SelfOrganised Critical (SOC) behaviour.

SOC generated considerable debate amongst earthquake forecasting researchers. The SOC state is considered to be a minimally-stable state, characterised by a high mean energy in the system with very small mean energy fluctuations (BAK et al., 1987). Events occur at random and are distributed according to a power-law sizedistribution. Assuming the earth's crust is in an SOC state, GELLER et al. (1997) argued that earthquakes cannot be predicted. In response, Wyss et al. (1997) cited a variety of evidence for precursory activity prior to catatrophic failure to argue that further research is required before earthquake forecasting is proven to be unfeasible.

One type of precursory activity which has driven the forecasting debate in recent years is that of accelerating seismic moment release. BuFE and VARNES (1993) demonstrated that the 1989 Loma Prieta, California earthquake was preceded by an interval of about $30-50$ years in which the rate of seismic activity accelerated. Employing cumulative Benioff strain release (square-root of the seismic moment release) as a measure of seismic activity, the researchers utilised an empirical time-tofailure relationship to make a retrospective forecast of the time-of-occurrence of the $M_{w}=6.9$ Loma Prieta earthquake with a precision of 2 months. The earthquake magnitude was predicted to be $M_{\text {pred }}=6.83$ (BUFE and VARNES, 1993). Subsequently, accelerating moment release has been identified prior to a considerable number of moderate to large earthquakes from a variety of tectonic settings. JAUME and SYKES (1999) presented a review of reported cases of accelerating moment release.

Power-law accelerations in energy release are an important feature of a class of dynamical systems known as Critical Point (CP) systems. In statistical physics, 
Renormalisation Group Theory was developed to describe CP systems. This theory identifies accelerating energy release as the signature of the progressive development of long-range correlations in a physical property of the system (SORNETTE and SAmmis, 1995). This concept of correlation evolution became the basis for the Critical Point Hypothesis (CPH) of earthquakes (SAleur et al., 1996). Under this hypothesis, an earthquake rupture may only occur when long-range stress correlations exist along the length of a fault. The progressive formation of such correlations is driven by tectonic loading and the redistribution of stress due to smaller earthquakes in the neighbourhood of the fault. Earthquake rupture destroys the long-range stress correlations, returning the fault to a state far-from-failure until a sufficiently correlated stress field is reformed via the action of subsequent, smaller earthquakes.

Renormalisation group theory and cellular automata have a number of features in common. The earthquake forecasting debate fueled considerable research employing both these techniques (see MAIN, 1996 for a review). The majority of automata studied were nearest-neighbour models; interactions only occur between cells and those adjacent to them. Weatherley et al. (2000) found that nearest neighbour automata may display behaviour in which the size of highly correlated regions increases prior to large events. However in these models, the formation of such correlated regions is driven predominantly by external (tectonic) loading rather than strain redistribution due to smaller to moderate events, as predicted by the CPH. SAmmis and Smith (1998) did identify correlation evolution (consistent with the $\mathrm{CPH}$ ) in an automaton consisting of a discrete fractal heirarchy of cells, including one macroscopically large cell which failed during the largest events.

Applying Damage Mechanics principles, XIA et al. (1999) developed a 1-D fibrebundle model displaying behaviour qualitatively similar to CP behaviour, known as Evolution Induced Catastrophy (WEI et al., 2000). In this model, strain energy from failed fibres was transferred to fibres within a local region whose size may be predefined or may depend upon the local pattern of damage. Unlike nearest neighbour models, the longer-range transfer of strain in the fibre-bundle model resulted in evolution qualitatively similar to $\mathrm{CP}$ behaviour.

KLEIN et al. (2000) examined a long-range automaton which is based upon a cellular automaton equivalent of the Burridge-Knopoff block-slider model (RUNDLE and JACKSON, 1997; RUNDLE and BROWN, 1991). KLEIN et al. employed a discretised stress Green's function with a $r^{-3}$ dependance to characterise the long-range interactions. Long-range springs join blocks to a large number of surrounding blocks and $r$ is defined as the distance between two blocks which are attached to oneanother. This model results in Gutenberg-Richter event size-distributions with a $b$ value of $b=1.5$, in agreement with a coarse-grained, mean-field description of the model derived by KLEIN et al. (2000). Scaling of events in these models is associated with nucleation near a mean-field spinodal rather than critical point fluctuations (KLEIN et al., 1997). 
Our motivation for designing the model presented in this paper, was to determine whether dynamical behaviour consistent with the $\mathrm{CPH}$ may be obtained in 2-D automata employing long-range transfer of strain energy from failed cells. A conceptual approach has been employed to design a model for the evolution of strain energy supported by contacting asperities within the plane of a fault. A 2-D grid of cells represents the contacting asperities. Each cell is predefined with a constant fracture energy, and has a variable strain energy. Tectonic loading of the fault is simulated by increasing the strain energy of all cells by a small amount each loading timestep. When the strain energy of a cell exceeds its fracture energy, the strain of the cell is transferred to cells within a square transfer region surrounding the failed cell.

An elastodynamic interaction function (similar to a Green's function) with a $r^{-p}$ dependance determines the amount of strain energy transferred to cells a distance $r$ from the failed cell. The form of the interaction function was chosen because linear elasticity predicts that, for a 2-D dislocation in a 3-D homogeneous elastic medium, the magnitude of the stress Green's function (T) decays as $T \sim r^{-3}$. Based upon this, one might expect $p=3$ is a good choice of interaction exponent (or perhaps $p=2$ for the 2-D case). However as mentioned by KLEIN et al. (2000), microcracks, fault gouge, and pore-fluids may mask the $r^{-3}$ interaction, resulting in effectively longerrange static stress interactions. In addition, crack propagation theory predicts $1 / \sqrt{r}$ dynamic stress concentrations at the rupture front of propagating cracks. MorA and PLACE (2002) report that correlation evolution can occur in particle-based elastodynamic systems, suggesting that $p \leq 1$ may be more appropriate for the interaction exponent. Furthermore, for elasto-plastic cases a $\log r$ term may appear. Hence, it is unclear on physical grounds what is the appropriate exponent for the interaction function. For this reason, we implement the model for an arbitrary choice of interaction exponent and then perform numerical experiments employing various values of the interaction exponent in the range $0.2<p<3$.

It is important to emphasise that the elastodynamic interaction range as defined by the interaction function (or the stress Green's function) is not equivalent to the correlation range as defined in the $\mathrm{CPH}$. The $\mathrm{CPH}$ proposes that correlations in some physical property (stress, strain, strain energy, or other) evolve in the lead-up to a large event. This evolution is characterised by an increase in the average size of regions with correlated properties. Hence, it is often stated that the correlation range increases as a catastrophic earthquake becomes imminent. However, the elastodynamic interaction range defined by the stress Green's function of the Crust, is not expected to evolve in the lead-up to a large event. In our model, the interaction exponent is held constant during any given simulation and so, undergoes no evolution.

The results which follow testify that, even with a fixed elastodynamic interaction range, correlation evolution may occur prior to large events. It is apparent that the interaction exponent $(p)$ is a tuning parameter governing the mode of dynamical behaviour displayed by the model. Small interaction exponents $(p<1)$ correspond- 
ing to effectively long-range interactions, result in dynamics dominated by characteristic, large earthquakes. These earthquakes are preceded by accelerating strain energy release, associated with global evolution of strain energy correlations within the system. Systems with intermediate-range interactions $(1<p<1.5)$ appear to switch between this characteristic mode and a dynamical mode characterised by linear energy release and no significant correlation evolution. Linear energy release eventually dominates the dynamical behaviour of the model, as $p \rightarrow 2$. Implications of these results for theoretical seismology and earthquake forecasting research are discussed in the sections following the results.

\section{Model Description}

\section{Basic Features of the Model}

The model consists of a regular, square lattice containing $N^{2}$ cells. Each cell $i=1, \ldots, N^{2}$ is assigned a variable, scalar strain energy $\varepsilon_{i}$ which is initially set to zero. In addition, each cell is also assigned a constant, scalar fracture energy, $\varepsilon_{f i}$ in the range $0.1<\varepsilon_{f i}<1.0$.

The fracture energies of cells are constrained to a statistical fractal distribution with a dimension, $D=2.3$. The statistical fractal is generated using a well-known Fourier filtering technique (TurCOTTE, 1997). The value of the fractal dimension was chosen because "fractal statistics with a fractal dimension near 2.2 are a good approximation for many geological processes" (HuANG and TURCOTTE, 1988). This is based upon detailed studies of the earth's topography.

The strain energy of all cells is periodically increased by an amount $\Delta \varepsilon$, known hereafter as the strain increment. The value of the strain increment is determined dynamically as the minimum strain energy required to fail at least one cell in the model. Failure of a cell occurs when the strain energy of the cell equals or exceeds its fracture energy, i.e., $\varepsilon_{i} \geq \varepsilon_{f i}$. Strain redistribution from failed cells is described in detail below.

An external loading mechanism of this form is often employed in automata to simulate slow tectonic loading in the models. Use of a variable strain increment $(\Delta \varepsilon)$ motivates the introduction of a model timescale $\left(t_{l}\right)$ which is proportional to the strain increment. The model time $\left(t_{\ell}(n)\right)$ of the $n$-th loading increment is given by

$$
t_{\ell}(n)=\sum_{j=1}^{n} K \Delta \varepsilon(j)
$$

Defined in this manner, the model time ensures a constant rate of external loading in accord with tectonic loading of earthquake faults. Since model units are arbitrary, we set $K=1$. Temporal statistics such as energy release are analysed with respect to model time rather than the number of loading timesteps. 


\section{Strain Redistribution}

The strain energy of a failed cell is redistributed to neighbouring cells in the grid. In classical cellular automata, the strain energy of failed cells is transferred to the 4 (or 8) nearest (or next nearest) neighbours in the grid. Redistribution of strain energy may trigger the failure of neighbouring cells. These cells in turn, redistribute their strain energy possibly triggering further cell failures. It is this mechanism of cascading ruptures which results in power-law event-size statistics similar to those of natural earthquakes.

The models examined in this paper employ a strain redistribution mechanism which is a generalisation of the classical nearest neighbour mechanism. Unlike classical cellular automata, the strain energy from failed cells is redistributed to all cells within a square region $(s \times s)$ surrounding the failed cell. This region is called the transfer (or interaction) region. The fraction of the released strain energy transferred to a given cell $k\left(=1, \ldots, s^{2}\right)$ is a function of the Cartesian distance of cell $k$ from the failed cell at $\left(x_{f}, y_{f}\right)$. To achieve this, each cell $(k)$ within the transfer region is assigned an interaction coefficient, $I_{k}$ :

$$
I_{k}=\frac{r_{k}^{-p}}{I_{o}},
$$

where $p$ is the interaction exponent. If the strain energy of the failed cell is $\varepsilon_{f}$, then the strain energy transferred to cell $k$ is given by $\varepsilon_{f} I_{k}$. The normalisation constant, $I_{o}=\sum_{k=1}^{s^{2}-1} r_{k}^{-p}$ ensures that strain energy is conserved during strain redistribution i.e.,

$$
\sum_{k=1}^{s^{2}-1} I_{k}=1 .
$$

The numerical experiment described in the following section consists of a parameter space investigation in which the interaction exponent is varied in the range $0.2<p<3$. We chose to vary the interaction exponent because, as discussed in the Introduction, it is unclear on physical grounds what is an appropriate value for the interaction exponent. As the exponent decreases, the rate of decay of the interaction function decreases. In terms of strain redistribution, this translates into a greater fraction of the released strain being transfered to larger distances in the model (as the exponent $p$ decreases). In other words, the effective range of elastodynamic interactions increases, as $p$ decreases.

In the simulations described below, only non-zero values of $p$ are employed. The transfer region size $(s)$ is chosen so that the minimum strain redistributed is small compared with the minimum cell fracture energy $(0.1)$ in most simulations. Under such circumstances, the finite size (and shape) of the transfer region has a negligible impact upon strain redistribution; the interaction function determines the spatial redistribution of strain energy. 


\section{Cell Healing and Dissipation}

In order to simulate seismic cycles, a method for healing failed cells must be specified. During the design phase of the model, a variety of different cell healing mechanisms were tested. These included mechanisms in which failed cells rapidly heal and may support strain energy transferred from the rupture front, and slow-healing mechanisms in which failed cells cannot support strain until after the rupture has completed. Rapid healing of cells resulted in two features which the authors considered undesirable on physical grounds. The first is that cells may fail multiple times during a given rupture. While earthquake rupture is a complex phenomenon, it is unlikely that a given portion of the rupture surface re-activates multiple times during earthquake rupture.

The second undesirable feature of rapid healing mechanisms is that very small mean energy fluctuations often result. Past research (WEATHERLEY et al., 2000; JAUMÉ et al., 2000) has shown that CP-like behaviour is most evident in models displaying large mean energy fluctuations. Linear energy release and no significant global correlation evolution corresponds to models displaying very small mean energy fluctuations. Thus, motivated by the physical grounds outlined above and a desire to simulate critical point behaviour, we employ the following slow-healing mechanism.

The strain energy of a failed cell is reduced to zero and all of the stored strain is redistributed within the cell's transfer region i.e., no bulk dissipation of strain energy is employed in this model. Once failed, a cell cannot support strain energy until completion of the rupture which failed that cell; cells do not fail multiple times during a given rupture. Strain energy transferred to failed cells is dissipated from the model. As a result, subsequent to any given rupture, the strain energy of cells within the rupture zone is zero. This is somewhat analogous to a frictional instability in which the stress of slipping fault patches decreases to a constant, residual level regardless of the frictional strengths of the patches.

One possible interpretation for the dissipative healing mechanism employed, is that the energy dissipated by failed cells represents the strain energy lost due to other processes within the fault zone such as frictional heating, and pore-fluid depressurisation. Perhaps one can argue that such a mechanism is qualitatively justified on physical grounds. The results which follow show that this dissipative healing mechanism results in long-term, stable dynamical behaviour including global correlation evolution consistent with the Critical Point Hypothesis.

As in many previous automata, open boundary conditions are employed. All strain energy transferred across external boundaries is dissipated from the model. The transfer and dissipation of strain energy across model boundaries may be considered analogous to the loss of strain energy from a fault zone due to transport of strain (during rupture) to another portion of the fault zone outside the model region. While some may argue for closed boundaries, in the authors' experience this 
often leads to highly conservative models which evolve towards a state of SOC quite unlike the dynamics of the models described here, unless a large portion of released strain $(>50 \%)$ is lost via bulk dissipation.

\section{Numerical Experiment and Empirical Results}

The model specified above is arbitrary in the choice of interaction exponent $(p)$ and transfer region size $(s)$. In a previous study of long-range automata the transfer region size was varied in order to characterise the change in system behaviour as the number of cells which receive strain energy from failed cells is increased (KLEIN et al., 2000). These simulations employed a constant interaction exponent (usually $p=3$ ) in a model with a fast healing, bulk dissipation mechanism. Under these conditions, increasing the transfer region size is considered equivalent to increasing the interaction range within the system (KLEIN et al., 2000).

As discussed earlier, we wish to explore a hypothetical 'parameter space' of models with differing interaction exponents $(p)$. In this we hope to determine whether the evolution of correlated regions of strain energy is affected by the fixed, effective elastodynamic interaction range within the system. Thus, for each simulation we employ a fixed transfer region size $s=59$ and vary the value of $p$.

The transfer region size is chosen so that the finite size of the region does not result in significant truncation of the interaction function (Equation 1) for simulations employing an interaction exponent in the range, $0.5<p<3$. Simulations employing interaction exponents in the range $0.2<p<0.5$ are also considered, however these simulations suffer some truncation by the square transfer region as the interaction exponent, $p \rightarrow 0.2$. This truncation gives rise to obvious undesirable features in the dynamical behaviour for simulations employing very small exponent values.

The same fracture energy distribution is employed for all simulations. Preliminary studies with various fracture energy distributions and various fractal dimensions have shown that the dynamical behaviour illustrated here, is relatively robust with respect to the choice of fracture energy distribution and range of fracture energies.

A total of 50 simulations were performed, each with a unique interaction exponent chosen from the set $p \in\{0.2+0.05 i \| i=0,1, \ldots, 56\}$. In each simulation, $10^{6}$ loading timesteps were computed, resulting in a synthetic earthquake catalogue consisting of $10^{6}$ events. The simulations were performed on a SGI Origin 3800 supercomputer with $16 \times 400 \mathrm{MHz}$ R 12000 CPUs. To compute $10^{6}$ loading timesteps and record all relevent model data typically consumes one hour of computation time on a single CPU of this supercomputer. The results presented in the following subsections often involve considerable post-processing of the recorded model data. In particular, to compute the correlation function of the strain energy field for only 500 timesteps required approximately five hours of CPU time. This placed obvious restrictions upon the depth of the investigation into strain energy correlation evolution. 
In each of the following subsections, various aspects of the models and their dynamical behaviour are examined in detail. These serve to illustrate both the dynamical behaviour of the model for a given interaction exponent, and the change in dynamical behaviour as the interaction exponent is varied. A summary of the results, some discussion, and possible implications for seismology are discussed subsequent to the numerical results.

\section{Rupture Properties and Event Statistics}

Strain redistribution from failed cells may trigger the failure of nearby cells which in turn, trigger failure of other cells. In this fashion, a cascading chain reaction can result in the failure of a macroscopic portion of the model. The pattern of failed cells for a typical large event from a simulation with $p=1.2$ is shown in Figure 1a along with snapshots of the strain energy in the model immediately before (Fig. 1b) and subsequent to (Fig. 1c) this event. The regions where the most cells failed in the event correspond to the mostly highly strained regions prior to the event. It is also evident that large events are capable of failing regions with a relatively low strain prior to the events; provided these regions reside near a highly strained cluster of cells which fail during the large event.

The size of an event in an automaton simulation is often defined as the number of cells which fail during a single rupture cascade. This is sufficient if all cells have the same fracture energy (i.e., in a uniform automaton). However, for automata with heterogeneous fracture energies such as those considered here, a more appropriate definition of the event size $(E)$ is the sum of the strain energy redistributed from all failed cells during the rupture cascade. The event size includes all strain energy transferred to other cells in the model and the strain energy dissipated from the model during the event.

Seismologists became interested in cellular automata when BAK and TANG (1989) showed that even highly simplified automata give rise to complex dynamics resulting in a power-law event-size distribution not unlike the empirical Gutenberg-Richter distribution. Cumulative event-size distributions for simulations with various interaction exponents may be compared in Figure $2 \mathrm{a}$ and interval event-size distributions for simulations with $p=1,2,3$ are given in Figures $2 \mathrm{~b}-\mathrm{d}$, respectively.

It is apparent that simulations with a relatively large effective interaction range (small $p$ ) produce an overabundance of large events. This overabundance diminishes as the interaction range decreases. From the interval distributions, it is evident that a power-law (or Gutenberg-Richter) event-size distribution is obtained for $p \sim 2$. For this value of $p$, the slope of the interval distribution is approximately 1.5, the same value as the theoretical prediction by KLEIN et al. (2000) for their similar BurridgeKnopoff, long-range automata. For interaction exponents $p>2$, the interval eventsize distribution begins to roll-over, indicating that these simulations have an underabundance of moderate to large events. Little study of models with $p>2$ has 


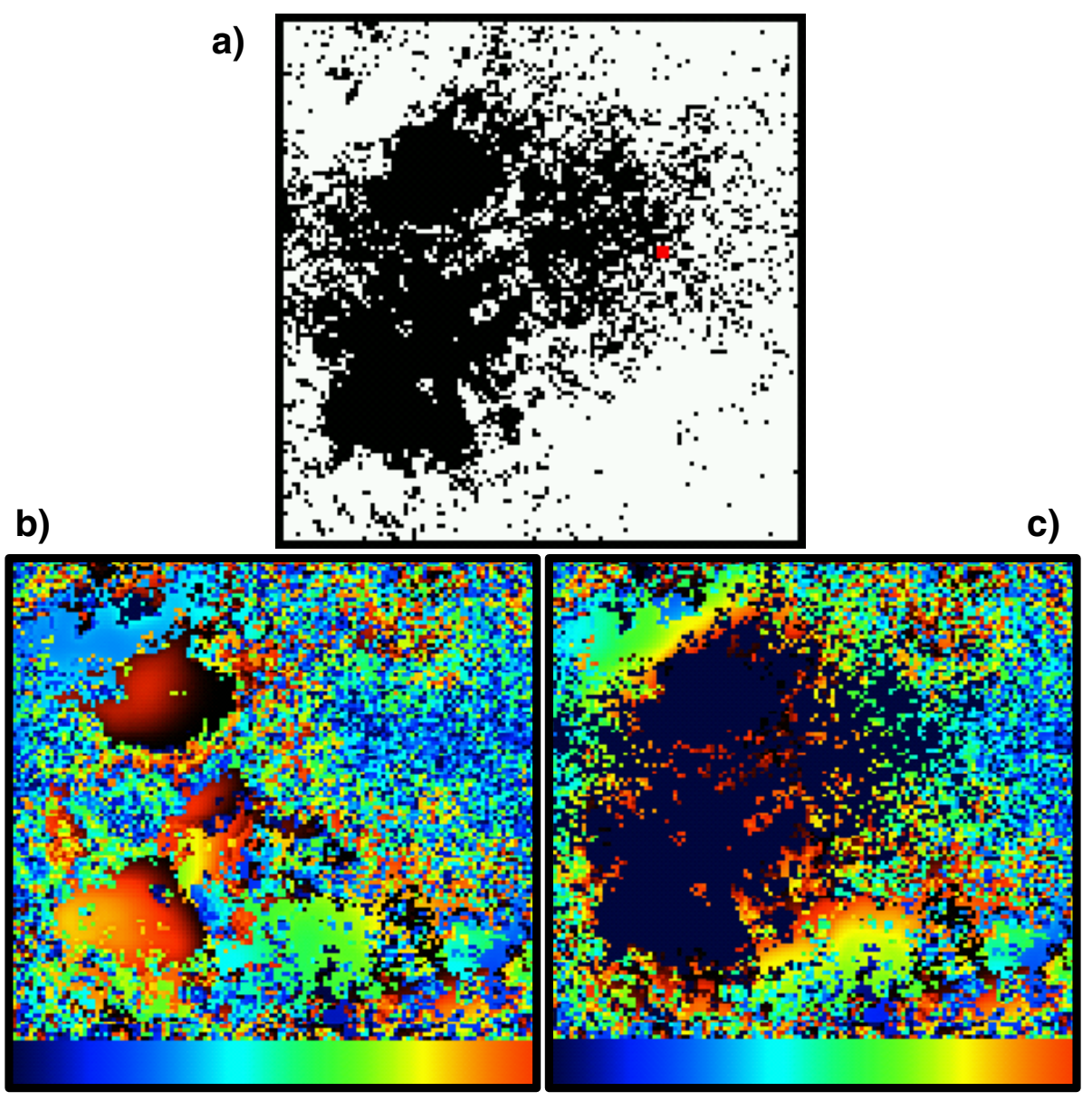

Figure 1

(a) Damage pattern for a large event in a simulation with $p=1.2$. The event (whose epicenter is denoted by a red square) failed a total of 5896 cells, releasing 3360.7 units of strain energy. Snapshots of the strain energy in the model (b) before and (c) after this event. The snapshots illustrate that prior to large events, there is considerable strain energy concentrated in the regions where the most cells fail. In the snapshots, hotter (red) colours represent the highest strain energies $(\sim 1.0)$ and cooler (blue) represents the lowest strain energies $(\sim 0.1)$.

been performed, although cursory investigation reveals a significant qualitative change in rupture dynamics. Such behaviour may be of interest to researchers in fields other than seismology.

\section{Energy Conservation and Temporal Fluctuations}

Each loading timestep, three processes alter the strain energy stored by cells in the model. These are the loading mechanism, strain redistribution during failure, and the 
a)

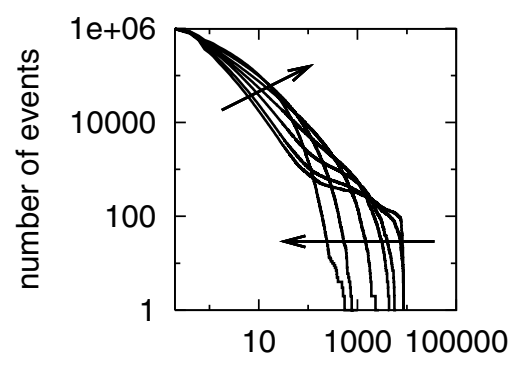

c)

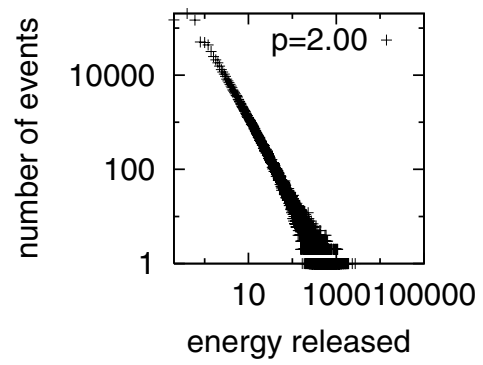

b)

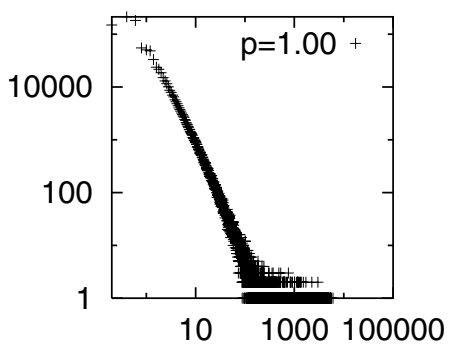

d)

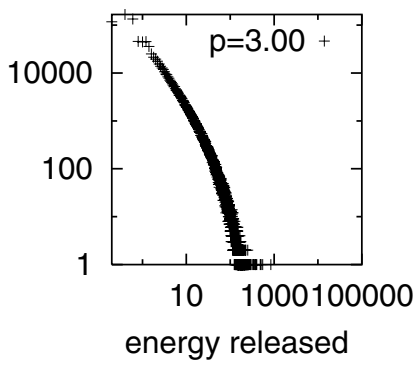

Figure 2

(a) Cumulative event-size statistics for simulations with a range of interaction exponents, $p \in[0.2,3.0]$. Arrows indicate the direction of increasing $p$. (b-d) Interval distributions for simulations with $p=1.0,2.0,3.0$, respectively. Notice that simulations with a longer effective interaction range $(p=1.0)$ tend to display an overabundance of large events. GR statistics with a slope of approximately 1.5 are obtained for $p=2.0$.

resultant energy dissipation. The total stored strain energy $\left(\varepsilon_{T}^{n}\right)$ at the end of the $n$-th timestep, may be expressed in terms of the total stored strain energy at the beginning of the $n$-th timestep (i.e., $\varepsilon_{T}^{n-1}$ ), the total increase in strain energy due to external loading $\left(\varepsilon_{L}^{n}\right)$, and the total energy dissipated $\left(\varepsilon_{D}^{n}\right)$ during the rupture. This relationship expresses the condition for energy conservation during a given loading timestep:

$$
\varepsilon_{T}^{n}=\varepsilon_{T}^{n-1}+\varepsilon_{L}^{n}-\varepsilon_{D}^{n} .
$$

Since the strain energy of each cell is incremented by $\Delta \varepsilon(n)$ in the $n$th timestep, $\varepsilon_{L}^{n}=N^{2} \Delta \varepsilon(n)$. Also, by the definition of the mean stored strain energy $\varepsilon_{T}^{n}=N^{2} \bar{\varepsilon}^{n}$. Using these substitutions, the energy conservation relation may be written in terms of the mean strain energy, the strain increment and the energy dissipated, i.e.,

$$
\bar{\varepsilon}^{n}=\bar{\varepsilon}^{n-1}+\Delta \varepsilon(n)-\frac{\varepsilon_{D}^{n}}{N^{2}}
$$

Evidently, the mean strain energy of the system increases monotonically whilst the energy dissipated is less than the energy increase due to external loading (i.e., 
$\left.\Delta \varepsilon(n)-\frac{\varepsilon_{D}^{n}}{N^{2}}>0\right)$. However, the mean energy will drop by an amount $\frac{\varepsilon_{D}^{n}}{N^{2}}-\Delta \varepsilon(n)$, if the energy dissipated in an event exceeds the loading energy. It may be concluded that the mean energy of the system should display a sawtooth behaviour, increasing gradually while only small events occur and dropping suddenly at the occurence of a large event.

Indicative time-series of the mean strain energy of the system are shown in Figures $3 \mathrm{a}-\mathrm{c}$ for $p=0.4,1.2,2.0$, respectively. These time-series consist of approximately 300,000 events and do not include transient behaviour due to the initial conditions; the time-series illustrate the long-term, stable dynamical behaviour of the model for various interaction ranges. The sawtooth mean energy evolution is particularly evident for the curves with the largest amplitude of fluctuations (Fig. 3a).

The temporal average of the mean strain energy at each timestep also displays a dependance upon the interaction exponent (Fig. 4a). Three regimes are apparent as the interaction exponent increases. The first regime $(p<0.5)$ contains models suffering truncation of the interaction function by the finite transfer region. The lower $\bar{\varepsilon}$ in this regime may be a reflection of the finite truncation however this is yet to be verified. During the second regime $(1 / 2<p \leq 2)$, the average mean energy remains relatively constant around $\bar{\varepsilon} \sim 0.268$. In the final regime $(p \rightarrow 3)$, the average mean energy rapidly decreases once more.

a)
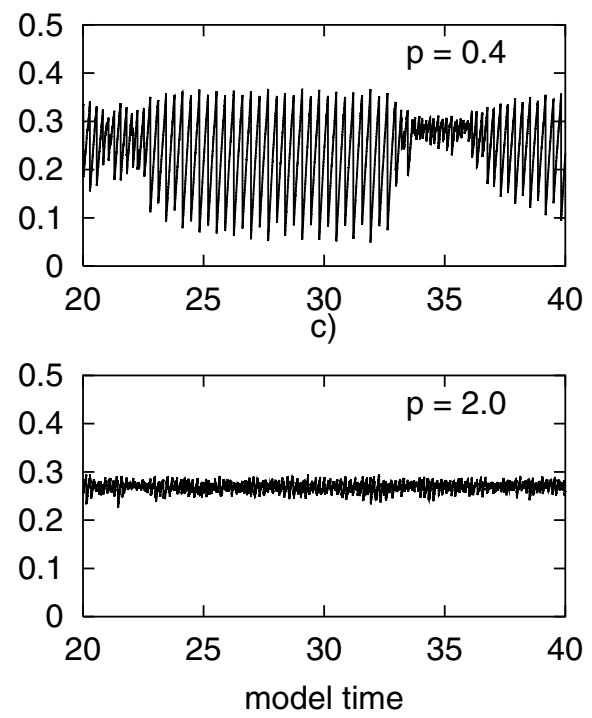

b)

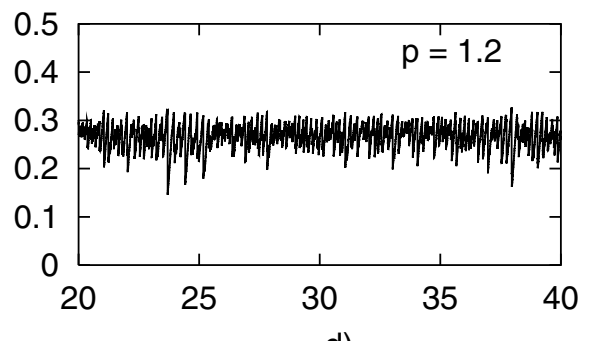

d)

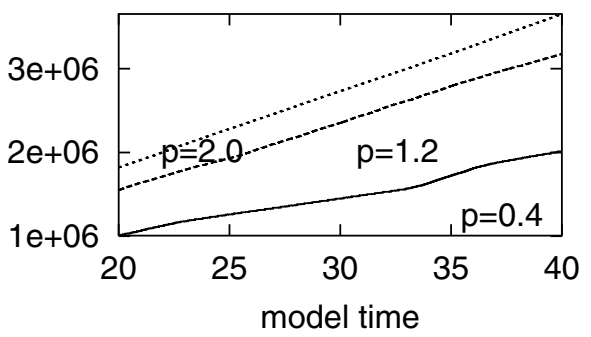

Figure 3

(a-c) Time-series of mean energy at the end of each timestep for approximately 300,000 timesteps of simulations with $p=0.4,1.2,2.0$, respectively. (d) Corresponding plots of cumulative energy release from the same simulations. For models with a longer effective interaction range, mode-switching behaviour is clearly evident, diminishing as $p \rightarrow 2.0$. 
a)

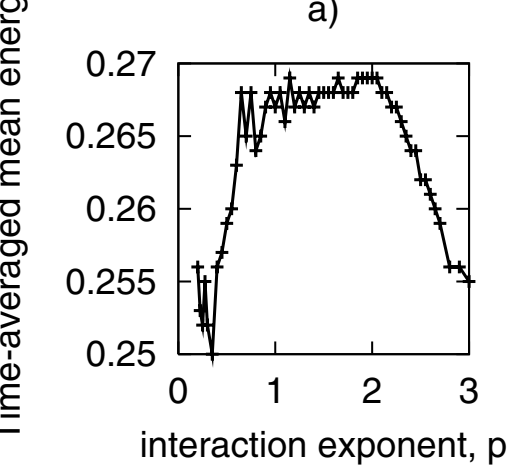

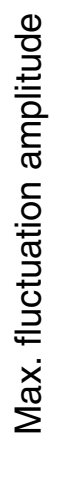

b)

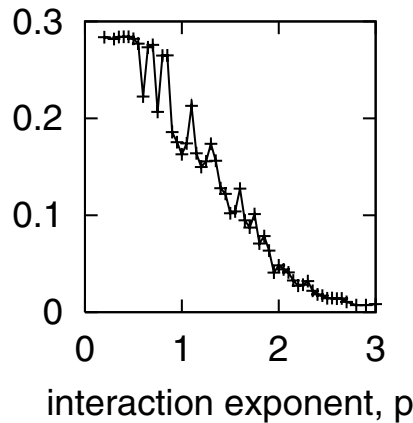

Figure 4

(a) Time-averaged mean energy for simulations with differing interaction exponents, $p$. (b) Estimates of the maximum amplitude of mean energy fluctuations for the same simulations.

In addition, a relationship between the amplitude of mean energy fluctuations and the interaction exponent, is clearly evident in Figures 3a-c. In an attempt to quantify this relationship, we have computed the maximum fluctuation amplitude for each simulation. This is achieved by recording the total energy dissipated $\left(\varepsilon_{D}^{n}\right)$ and the strain increment $(\Delta \varepsilon(n))$ each timestep. For all the simulations, the range of strain increment sizes is approximately $0<\Delta \varepsilon<10^{-3}$, after the initial 100,000 timesteps. Recognising that the majority of the smaller events occurring in the simulations do not dissipate an appreciable amount of energy, the range in energy dissipated per timestep is $0<\varepsilon_{D} \leq \varepsilon_{D \max }(p)$.

The maximum energy dissipated depends upon the maximum event size which is dependant upon the choice of interaction exponent (see Fig. 2). We take $\left(\varepsilon_{D \max (p)} / N^{2}\right)$ as a measure of the maximum fluctuation amplitude for a simulation with interaction exponent $p$ (c.f. Equation 2). The maximum fluctuation amplitude displays a decreasing trend when plotted versus the interaction exponent (Fig. 4b).

\section{Mode-switching Behaviour}

Time-series of cumulative energy release are given in Figure 3d. These correspond to the mean energy time-series in Figures $3 \mathrm{a}-\mathrm{c}$. For the simulation with $p=0.4$, two phases with a differing mean rate of energy release may be identified. Long, cyclic phases with large mean energy fluctuations and a relatively lesser mean rate of energy release are separated by relatively short intervals with a higher mean rate of energy release. The higher rate of energy release coincides with intervals in which the model displays relatively small mean-energy fluctuations (c.f. Fig. 3a). Such mode-switching behaviour is evident in all simulations with sufficiently large effective interaction range (with interaction exponents smaller than $p \sim 1.5$ ). However, as the interaction 
exponent increases, mode-switching becomes less obvious and linear energy release begins to dominate.

\section{Accelerating Rate of Energy Release Prior to Large Events}

Visual examination of cumulative energy release time-series revealed qualitative evidence for power-law accelerating sequences. This prompted an investigation to determine whether on average, the rate of energy release accelerates in the interval preceding large events in the model. We performed a simplified time-to-failure analysis of the cumulative energy release to determine the probability that a macroscopically large event is preceded by an interval in which the rate of energy release accelerates. We were particularly interested in whether the system-wide (global) energy release accelerates in the lead-up to a large event.

A power-law time-to-failure relationship is fit to the cumulative energy release in the interval preceding the 500 largest events in each of a selection of simulations. Following the methodology of BUFE and VARNES (1993), a power-law time-tofailure relationship of the following form is fit to energy release prior to large events:

$$
\sum_{t=\left(t_{f}-\Delta t\right)}^{t<t_{f}} E(t)=A+B\left(t_{f}-t\right)^{-m}, \quad(m>0)
$$

where $t_{f}$ is taken as the model time at which the large event occurs and $A, B$ are empirical constants. The interval $(\Delta t)$ and the power-law exponent $(m)$ are varied in the ranges $0.1<\Delta t<0.4$ and $0.1<m<1.0$, and a goodness-of-fit parameter $(\mathscr{C})$ is computed for each choice of interval length and exponent value. A minimum interval length of $\Delta t=0.1$ was imposed to ensure that all sequences contained at least 100 events.

The goodness-of-fit $(\mathscr{C})$ is defined as the ratio of the RMS error of the power-law fit to the RMS error of a linear fit for the same time interval. This parameter is chosen to maintain consistancy with past researchers examining natural accelerating seismic energy sequences (BUfE and VARnES, 1993). The fit with the smallest $\mathscr{C}$ is selected as the best power-law fit to the cumulative energy release for the given event, and the interval length, power-law exponent, and goodness-of-fit is recorded.

In the analysis, frequency histograms of the number of fits with given fit exponents $(m)$ and interval lengths $(\Delta t)$ were examined in addition to histograms of goodness-of-fit such as those of Figure 5. These histograms illustrate the general trend observed in the analysis: power-law fits to energy release are obtained more frequently in simulations with smaller values of $p$ (i.e., with effectively longer-range elastodynamic interactions). Linear energy release is referred for simulations with a shorter interaction range. 

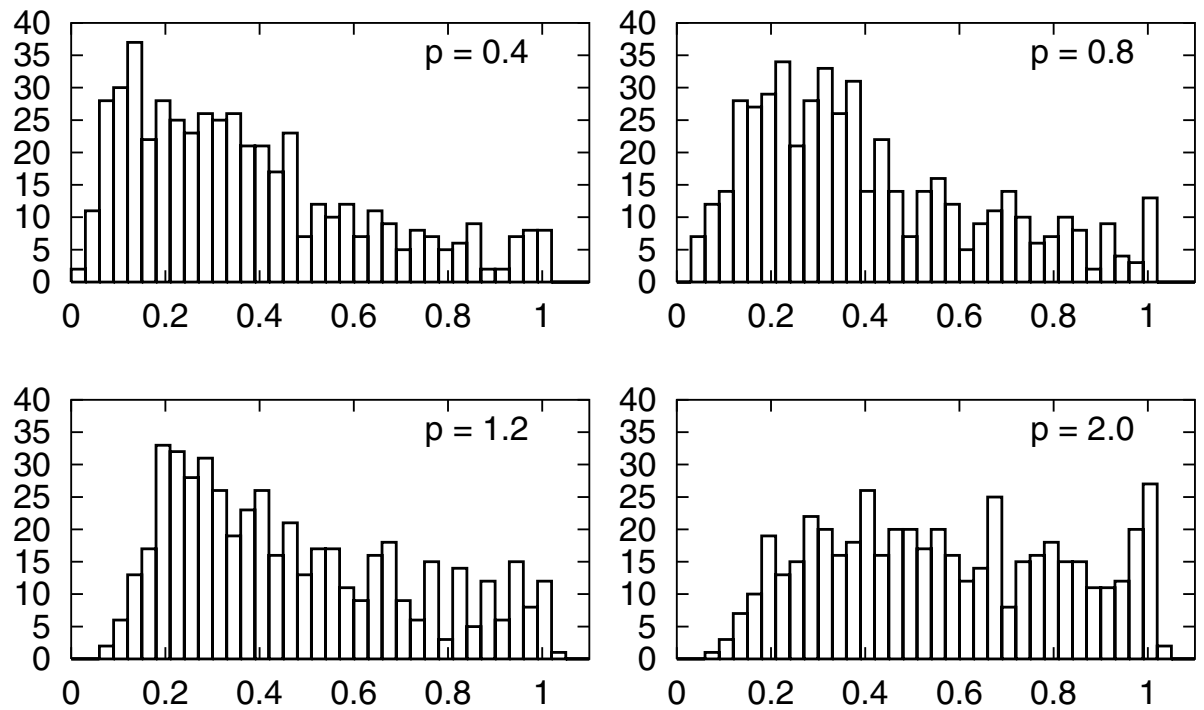

Figure 5

Frequency histograms of the number of power-law fits with a given goodness-of-fit, $\mathscr{C}$ for simulations with the given interaction exponent values. For small values of $p$, a large fraction of fits have $\mathscr{C}<1$ indicating good power-law fits to energy release sequences. This population is progressively replaced by predominantly linear energy release sequences, as $p \rightarrow 2$.

Examples of power-law fits to energy release are given in Figure 6. Corresponding linear fits to these sequences are provided for comparison. Quite reasonable power-law accelerating sequences are picked by the regression algorithm for simulations with $p=0.4$ and $p=1.2$. Second-order fluctuations in the data are reminiscent of log-periodic corrections proposed to exist in systems displaying discrete scale invariance (SAleur et al., 1996). The statistical fractal employed in these models has the property of discrete scale invariance.

Figure $6 \mathrm{c}$ illustrates a typical power-law fit to long energy release sequences in a simulation with $p=2$. On average, energy release is near-linear. However one might argue there is an appearance of higher-order log-periodic fluctuations in energy release prior to large events even for $p=2$. A possibility worth exploring is that large events in models with $p=2$, might be predicted if a higher-order log-periodic correction were applied to Equation (3). However this is simply conjecture at present; confirmation would require further research outside the scope of this investigation.

\section{Evolution of Strain Energy Correlations}

A selection of simulations were rerun and a sequence of strain energy snapshots were recorded during the last 3000 timesteps prior to the 50 largest events in each simulation. This sequence spans a time interval of approx. 0.2 model time units prior 
Power law fit to cumulative energy release

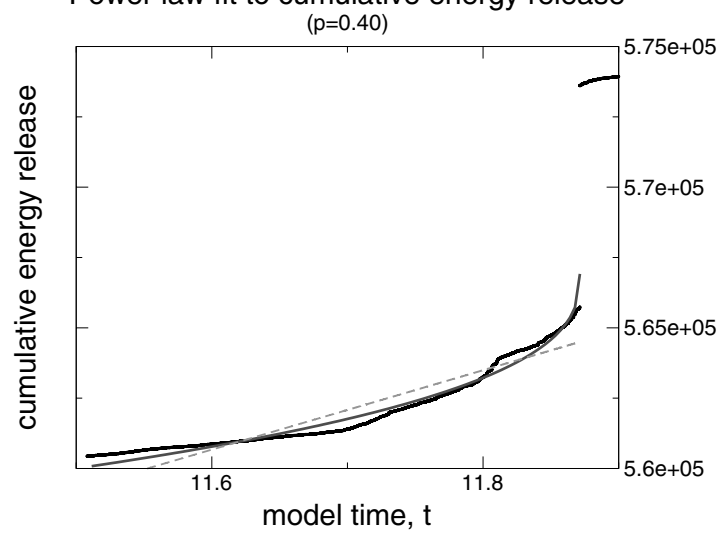

Power law fit to cumulative energy release

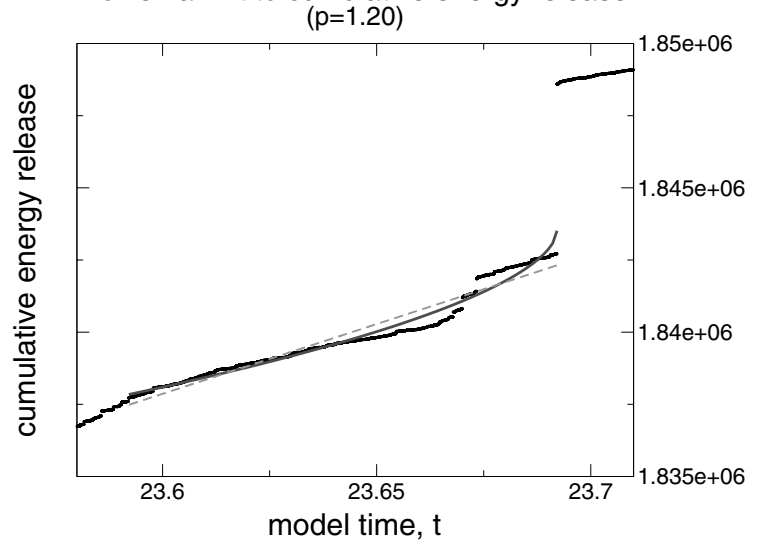

Power law fit to cumulative energy release

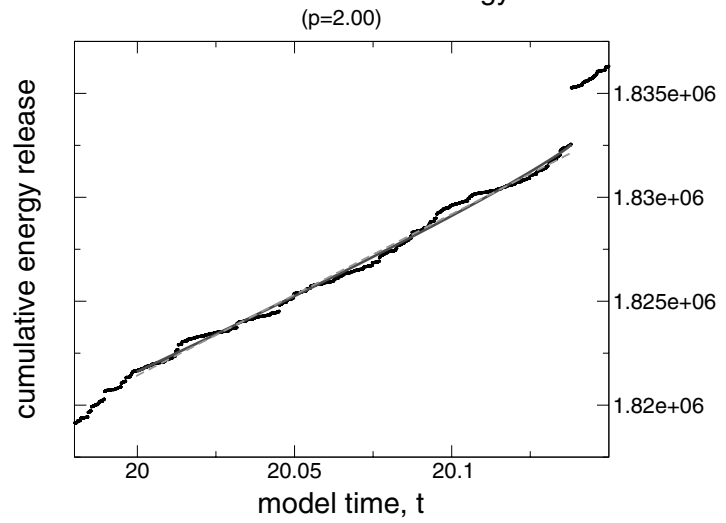


to each large event. A selection of such snapshot sequences are given in Figure 7. These correspond to the fitted event sequences in Figure 6.

During the accelerating sequences, small events occuring predominantly in the weak region (top, right) of the model redistribute strain into a broad, strong region (left) of the model. When the mean strain energy within this broad region reaches a sufficient level, a large event is triggered. Often the smaller events may trigger intermediate, moderate-sized events which fail only a portion of the highly strained region, prior to the large event. The evolution of the strain energy during such accelerating sequences is suggestive of the evolution expected if spatial strain correlations in the model evolve in a fashion consistent with the $\mathrm{CPH}$.

This is confirmed by performing a correlation function analysis of the strain energy field using the strain energy snapshots. The correlation function $C(r, t)$ of the strain energy field is defined as the following:

$$
C(r, t)=\frac{\left\langle\left(\varepsilon_{i}(t)-\bar{\varepsilon}(t)\right)\left(\varepsilon_{j}(t)-\bar{\varepsilon}(t)\right)\right\rangle}{\left\langle\left(\varepsilon_{i}(t)-\bar{\varepsilon}(t)\right)^{2}\right\rangle}
$$

where $r=\sqrt{\left(x_{j}-x_{i}\right)^{2}+\left(y_{j}-y_{i}\right)^{2}}$ and $\langle\cdots\rangle$ represent the average over all pairs of cells a distance $r$ apart. For the $128 \times 128$ model employed here, the range of distance scales is $0 \leq r<182$. The discrete contributions to the correlation function due to each cell-pair are linearly interpolated onto this range to produce a correlation function $C(r)$ for each strain energy snapshot. We stress that the correlation function so computed is the global strain energy correlation function of the system. Correlation evolution analysis for subsets of the model region were not performed in this investigation.

The denominator of the correlation function normalises $C(r)$ to remove any dependance upon the mean strain energy $(\bar{\varepsilon}(t))$. This is required because, as we have seen, the mean energy of the system increases on average prior to large events. Without normalisation, an apparent evolution of the correlation function may simply reflect the mean energy evolution, without any increase in the degree of correlations at longer range; the specific mechanism proposed by the $\mathrm{CPH}$.

During the analysis, individual energy release sequences were compared with corresponding snapshots and correlation functions. It was noticed that motion of the zero-crossings of $C(r, t)$ was related to the progressive growth of a broad, highly correlated region, prior to macroscopic events in the models. Space limitations precluded an in-depth analysis of this relationship.

Figure 6

Three power-law time-to-failure fits to cumulative energy release for three sequences indicative of those from simulations with $p=0.4,1.2,2.0$. The solid line represents the power-law fit and the dashed line is the best-fit linear approximation to the cumulative energy release data. 


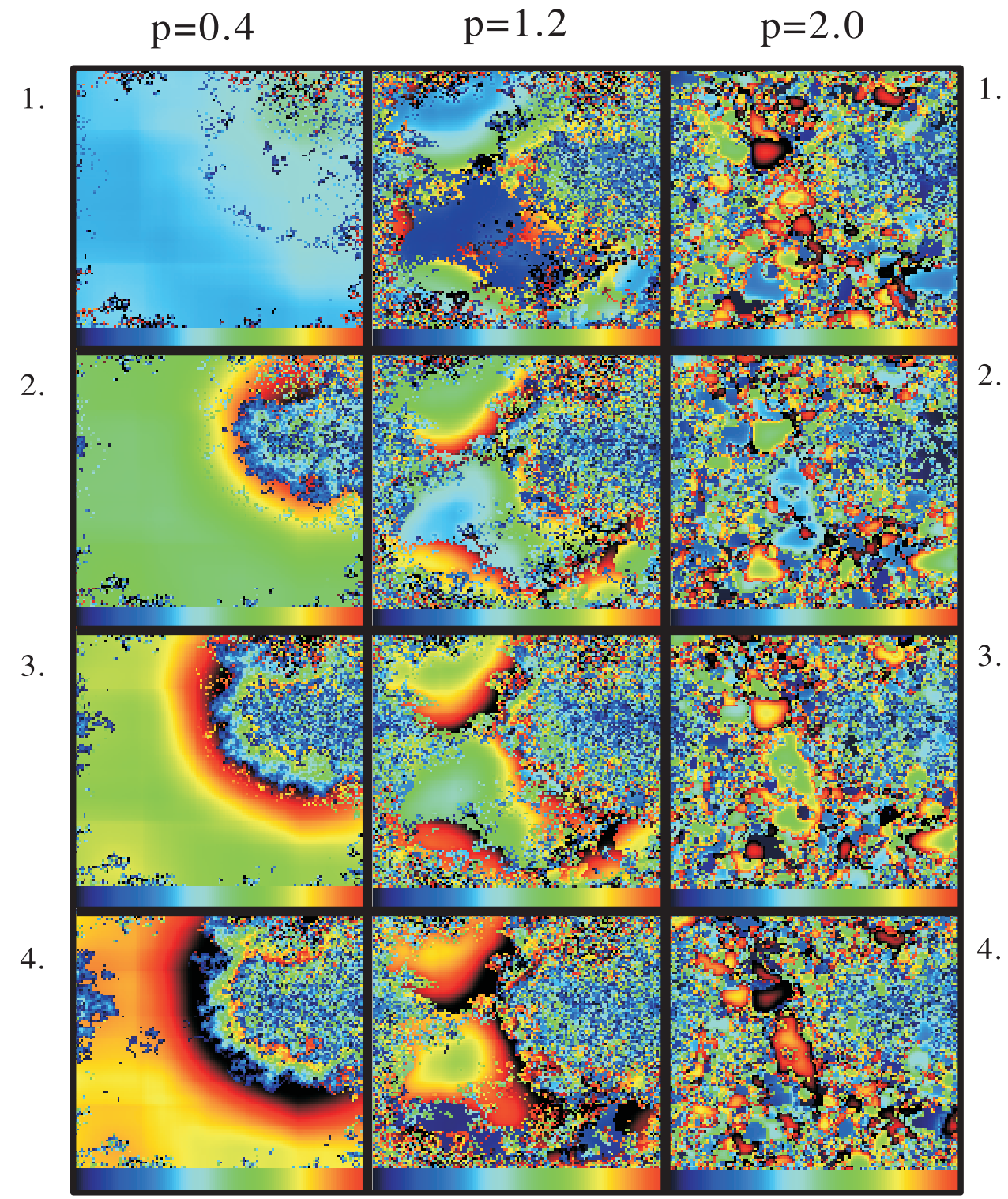

Figure 7

A sequence of four strain energy snapshots corresponding to the power-law fits in Figure 6 . The first column is from a simulation with $p=0.4$, the second column is obtained for $p=1.2$, and the third is from a simulation with $p=2.0$. The colour scale is the same as Figure 1 .

Figure 8 attempts to illustrate the relative amount of correlation evolution which occurs in simulations with differing elastodynamic interaction ranges (differing $p$ ). The curve for each $p$ value is obtained by first computing the mean correlation function immediately prior to the 50 largest events, and also 0.2 model time units 


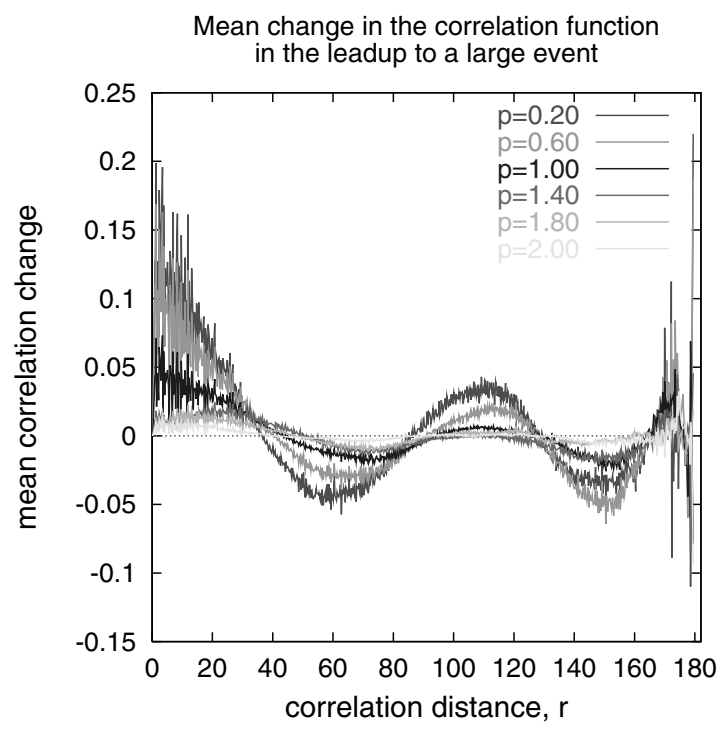

Figure 8

The mean change in correlations at each scale $r$ in the lead-up to a large event, for various values of the interaction exponent. Global evolution of strain energy correlations occur prior to large events in simulations with longer-range elastodynamic interactions $(p \sim 1)$. However little or no, correlation evolution precedes large events as $p \rightarrow 2$.

prior to these events. The mean correlation function from the earlier time is subtracted from the mean correlation function immediately prior to failure to give a mean change in correlations in the interval leading up to the largest 50 events.

It is quite evident in Figure 8 that there is evolution in the range $0.2<p<2$ and the amplitude of the mean correlation change increases as the effective interaction range increases (i.e., as $p \rightarrow 0$ ). On the other hand, a small effective interaction range $(p \sim 2)$ results in little or no global correlation evolution prior to the largest 50 events. It is apparent from these results that correlation evolution consistent with the $\mathrm{CPH}$ accompanies accelerating energy release sequences prior to large events in models with effectively long-range elastodynamic interactions $(p \sim 1)$. However, linear energy release sequences are accompanied by no significant global correlation evolution $(2 \leq p<3)$.

\section{Discussion}

The conceptual design of this model makes it difficult to claim that these models are a good representation of the physics occurring within the plane of a natural fault. However, the behaviour of these models may be somewhat analogous to natural earthquake faults, if the constitutive friction law governing fault rupture results in 
small events which predominantly move strain energy around within the fault region and large events which remove a considerable fraction of the stored strain energy from the region. With these characteristics, natural earthquake faults may display behaviour not too dissimilar to the behaviour described above.

The implications for prediction of large events in these models should be obvious: large events may only be forecast reliably when the system resides in the accelerating mode. In this case, a forecast of the time of occurrence of a large event may be made by fitting the cumulative energy release to a power-law time-to-failure relationship, or by periodically computing the strain energy correlation function, or both. On the other hand, large events occurring during the linear mode appear to display no systematic, global precursory behaviour upon which to base a forecast. The phenomenon of mode-switching indicates that, within any given simulation, both predictable and unpredictable events occur.

Similar mode-switching behaviour has been identified in other earthquake analogue models including a 3-D regional lithospheric model consisting of an upper brittle zone with a damage rheology, over a Maxwell viscoelastic substrate (BENZION et al., 1999). In agreement with the results here, one mode was characterised by Gutenberg-Richter scaling, while the other mode was charaterised by a characteristic earthquake distribution. According to BEN-ZION et al., "the mode-switching phenomenon may also exist in responses of other systems with many degrees of freedom and nonlinear dynamics."

The stimulus which prompts a system with long-range interactions to switch from one stable mode to the other is unknown. However, it is thought that heterogeneity in the strain energy field is involved. In this model, accelerating energy release and correlation evolution appears to occur when the strain energy field is relatively smooth at small scales. Conversely, large small-scale heterogeneity in the strain energy field promotes linear energy release. Further research is planned to measure the degree of small-scale strain heterogeneity as a simulation approaches a switch between one type of behaviour and the other. It is hoped that the evolution of strain heterogeneity in the system may herald the transition between the different dynamical modes.

\section{Conclusions}

The model described in this paper displays two distinctly different stable dynamical modes. The first mode is characterised by small mean energy fluctuations, linear energy release, and a lack of global correlation evolution. The second mode is characterised by large mean energy fluctuations, and large events are preceded by accelerating energy release associated with evolution of the strain energy correlation function. It is apparent from the results presented that the dynamical behaviour of the model undergoes a transition from one stable mode to another, as the interaction 
range is varied. A small interaction range $(p \geq 1.5)$ results in dynamics dominated by the first, linear mode whilst models with larger interaction ranges $(p<1.5)$ appear to mode-switch between the accelerating mode and the linear energy release mode. It is apparent that global correlation evolution consistent with the $\mathrm{CPH}$ occurs when the model resides in the second mode, suggesting that forecasts of macroscopic events are feasible under these circumstances.

Evidence for accelerating seismic moment release prior to a number of natural earthquakes has been reported, as has a few events which were not preceded by any acceleration in the rate of seismic moment release (JAUMÉ, 2000). These observations suggest that dynamical behaviour similar to that of the model, may be occurring within the earth's crust. In this case, earthquake prediction may be feasible only for some large earthquakes but not for others. Further research with models such as these may help to identify observable consequences of being in one mode or another, so that the dynamics and stress state of the earth's crust may be inferred for a given region, and "predictable" earthquakes may be forecast reliably.

\section{Acknowledgments}

This research was supported by the Australian Research Council, the University of Queensland, the Special Funds for Major State Basic Research Project and the National Natural Science Foundation of China (Grant No. 19732060 and No. 19972004), and the ARC IREX ACES International Visitors Program. The authors wish to thank W. Klein and Y. Ben-Zion for insightful discussions and advice during this research project. Thanks also to the reviewers for constructive criticism of the original manuscript.

\section{REFERENCES}

BAK, P. and TAng, C. (1989), Earthquakes as a Self-organised Critical Phenomenon, J. Geophys. Res. 94, B11, 15,635-15,637.

Bak, P., TAng, C., and Wiesenfeld, K. (1987), Self-organised Criticality: An Explanation of 1/f Noise, Phys. Rev. Lett. 59, (4), 381-384.

Ben-zion, Y., Dahmen, K., Lyakhovsky, V., Ertas, D., and Agnon, A. (1999), Self-Driven Mode Switching of Earthquake Activity on a Fault System, Earth Planet. Sci. Lett. 172, (1-2), 11-21.

Bufe, C. G. and VArnes, D. J. (1993), Predictive Modelling of the Seismic Cycle of the Greater San Francisco Bay Region, J. Geophys. Res. 98, (B6), 9871-9883.

Geller, R. J., Jackson, D. D., Kagan, Y. Y., and Mulargia, F. (1997), Enhanced: Earthquakes Cannot be Predicted, Science 275, (5306), 1616.

Gutenberg, B. and Richter, C. F. (1956), Magnitude and Energy of Earthquakes, Ann. Geofis. 9, 1.

Huang, J. and Turcotte, D. L. (1988), Fractal Distributions of Stress and Strength and Variations of b-value, Earth and Planet. Sci. Lett. 91, 223-230.

Jaumé, S. C. and Sykes, L. R. (1999), Evolving Towards a Critical Point: A Review of Accelerating Seismic Moment/Energy Release Prior to Large and Great Earthquakes, Pure Appl. Geophys. 155, $279-305$. 
Jaumé, S. C., Weatherley, D., and Mora, P. (2000), Accelerating Seismic Energy Release and Evolution of Event Time and Size Statistics: Results from Two Heterogeneous Cellular Automaton Models, Pure Appl. Geophys. 157, 2209-2226.

Klein, W., Rundle, J. B., and Ferguson, C. D. (1997), Scaling and Nucleation in Models of Earthquake Faults, Phys. Rev. Lett. 78, (19), 3793-3796.

Klein, W., Anghel, M., Ferguson, D., Rundle, J. B., and SÁ martins, J. S. (2000), Statistical analysis of a model for earthquake faults with long-range stress transfer. IN Geocomplexity and the Physics of Earthquakes, Geophysical Monograph 120, American Geophysical Union, 43-71.

Main, I. (1996), Statistical Physics, Seismogenesis, and Seismic Hazard, Rev. Geophys. 34, (4), 433-462.

Mora, P. and Place, D. (2002), Stress Correlation Function Evolution in Lattice Solid Elastodynamic Models of Shear and Fracture Zones and Earthquake Prediction, Pure Appl. Geophys., 159, 2413-2427.

Rundle, J. B. and Brown, S. R. (1991), Origin of Rate-dependence in Frictional Sliding, J. Statis. Phys. 65 , 403-412.

Rundle, J. B. and JACKSOn, D. D. (1997), Numerical Simulation of Earthquake Sequences, Bull. Seismol. Soc. Am. 67, 1363-1377.

Saleur, H., Sammis, C. G., and Sornette, D. (1996), Discrete Scale Invariance, Complex Fractal Dimensions, and Log-periodic Fluctuations in Seismicity, J. Geophys. Res. 101, (B8), 17,661-17,677.

Sammis, C. G. and Smith, S. W. (1998), Seismic Cycles and the Evolution of Stress Correlation in Cellular Automaton Models of Finite Fault Networks, Pure Appl. Geophys. 155, 307-334.

Sornette, D. and SAmmis, C. G. (1995), Complex Critical Exponents from Renormalisation Group Theory of Earthquakes: Implications for Earthquake Predictions, J. Phys. I France 5, 607-619.

Turcotte, D. L., Fractals in Geology and Geophysics (Cambridge Univ. Press, 2nd ed., 1997).

Weatherley, D., Jaumé, S. C., and Mora, P. (2000), Evolution of Stress Deficit and Changing Rates of Seismicity in Cellular Automaton Models of Earthquake Faults, Pure Appl. Geophys. 157, 2183-2207.

WeI, Y. J., XIA, M. F., KE, F. J., YIn, X. C., and BAI, Y. L. (2000), Evolution Induced Catastrophe and its Predictability, Pure Appl. Geophys. 157, (11/12).

Wu, Z. L. (2000), Frequency-size Distribution of Global Seismicity Seen from Broadband Radiated Energy, Geophys. J. Int. 142, 59-66.

Wyss, M., Aceves, R. L., and PARK, S. K. (1997), Cannot Earthquakes be Predicted?, Science 278, 487490.

XIA, M. F, BAI, J., KE, F. J., and BAI, Y. L. (1999), Sample-specificity and predictability of material failure. In 1st ACES Workshop Proceedings (Univ. of Queensland, 1999).

(Received February 20, 2001, revised June 11, 2001, accepted June 15, 2001)

To access this journal online:
(4D) http://www.birkhauser.ch 